\title{
Le bon tempo
}

\section{The Good Tempo}

\section{G. Lesur}

(C) Lavoisier SAS 2015

Les musiciens utilisent souvent ce terme, en général en italien, et il devient alors « tempo giusto ». Il correspond à une adéquation entre la musique telle qu'elle est écrite et telle qu'elle sonne dans le rythme choisi. À l'inverse, le tempo peut être trop rapide ou trop lent, ce qui dans le pire des cas fait perdre de la qualité, de la fluidité et donc son impact à la musique. L'appréciation du tempo est évidemment un peu subjective mais avec un peu d'expérience et d'écoute, même un néophyte se rend compte si le tempo est le bon.

Quel rapport me direz-vous avec Acta Endoscopica et la SFED ? Aucun, si ce n'est que c'est maintenant que j'ai choisi de quitter mes fonctions de rédacteur en chef d'Acta Endoscopica, fonction que j'occupe depuis deux ans, après avoir été rédacteur en chef adjoint à mon entrée au sein du conseil d'administration de la SFED en 2011. Il me semble avoir montré que l'on pouvait, avec un peu d'organisation et beaucoup de patience et de détermination, faire vivre une revue papier consacrée à l'endoscopie éditée en français. Quittant simultanément ce conseil d'administration, après un mandat unique de quatre ans durant lequel j'ai été secrétaire général adjoint durant deux ans, il me semble donc que le moment est le bon, le tempo juste, pour mettre fin à ses différentes activités.
En effet, je suis convaincu que nos sociétés savantes doivent se renouveler plus souvent et je m'applique donc ce à quoi je crois. Et quatre ans à travailler avec détermination à ce en quoi on croit, c'est déjà long. Si le monde politique faisait de même, on éviterait sans doute bien des copinages, qui sont sources d'immobilisme, et ces arrangements deviendraient plus difficiles et moins tolérés. Au moment où j'écris ces lignes, je ne connais pas le résultat des élections au conseil d'administration de la SFED, mais le nom des trois candidats au poste de représentant des praticiens hospitaliers de CHU que j'occupais me rassure ! Quel que soit mon successeur, il aura toute sa place à la SFED.

Je suis également heureux que la relève comme rédacteur en chef d'Acta Endoscopica soit confiée à Erwan Bories, un médecin gastroentérologue de talent qui exerce à l'Institut Paoli-Calmettes de Marseille, un centre de lutte contre le cancer. Spécialiste réputé, notamment en échoendoscopie et en endoscopie interventionnelle, il a toutes les qualités pour faire vivre et encore se développer Acta Endoscopica.

Je te souhaite donc, cher Erwan, beaucoup de succès à Acta Endoscopica. Et bonne route à la SFED et à sa revue, Acta Endoscopica.
G. Lesur $(\bowtie)$

Hôpital Ambroise Paré, 9, avenue Charles de Gaulle, F-92104

Boulogne-Billancourt cedex

e-mail : gilles.lesur@apr.aphp.fr 